\title{
Vestibular hypersensitivity to clicks is characteristic of the Tullio phenomenon
}

\author{
J G Colebatch, B L Day, A M Bronstein, R A Davies, M A Gresty, L M Luxon, \\ J C Rothwell
}

\begin{abstract}
Objectives-The frequency of pathologically reduced click thresholds for vestibular activation was explored in patients with the Tullio phenomenon (sound induced vestibular activation).

Methods-Seven patients (eight affected ears) with symptoms of oscillopsia and unsteadiness in response to loud external sounds or to the patient's own voice were examined. In all but one patient, vestibular hypersensitivity to sound was confirmed by the fact that eye movements could be produced by pure tones of $110 \mathrm{~dB}$ intensity or less. Conventional diagnostic imaging was normal in all cases and three of the patients had normal middle ears at surgical exploration. Thresholds for click evoked vestibulocollic reflexes were compared with those of a group of normal subjects. Galvanic stimulation was used as a complementary method of examining the excitability of vestibular reflexes.

Results-All the patients showed a reduced threshold for click activation of vestibulocollic reflexes arising from the affected ear. Short latency EMG responses to clicks were also present in posterior neck and leg muscles, suggesting that these muscles receive vestibular projections. Galvanic stimulation produced a normal pattern of body sway in four of the five patients tested.

Conclusions-A pathologically reduced threshold to click activation ( $\leqslant 70 \mathrm{~dB}$ NHL (average normal hearing level)) seems to be a consistent feature of the Tullio phenomenon and a useful diagnostic criterion. This in turn is most likely to be due to an increased effectiveness of the transmission of sound energy to saccular receptors. Activation of these receptors probably contributed to the vestibular symptoms experienced by the patients. (F Neurol Neurosurg Psychiatry 1998;65:670-678)
\end{abstract}

Keywords: Tullio phenomenon; vestibular function tests; vestibular nerve; acoustic stimulation; vestibulocollic reflex

Correspondence to: Dr JG Colebatch,

Department of Neurology, Prince of Wales Hospital, High Street, Randwick, Sydney, NSW 2031,

Australia. Fax 006129382 2428 .

Received 27 February 1998 and in revised form

7 May 1998

Accepted 15 May 1998.

The Tullio phenomenon is a rare but remarkable clinical entity in which everyday loud sounds will activate the vestibular apparatus. Affected patients develop oscillopsia and unsteadiness in response to, for example, shouting, traffic noise or their own voices. Normally, despite its proximity to the cochlea, the vestibular apparatus is relatively insensitive to sound and it is only when extremely loud sounds are encountered, such as are achieved near jet engines or explosions, that there is evidence of vestibular activation. ${ }^{1-3}$ Various disease states have been reported to underlie the pathological sound sensitivity that occurs in the Tullio phenomenon, including dislocation of the stapes footplate, labyrinthine fistulas, endolymphatic hydrops, and dehiscence of the superior semicircular canal. ${ }^{4-7}$

We have recently reported a patient with the Tullio phenomenon in whom there was a pathological increase in her sensitivity to a new test of vestibular function using clicks. ${ }^{8}$ This finding is of particular importance for various reasons, not least being that conventional tests of vestibular function in these patients show no consistent abnormality. Sound sensitivity does not necessarily imply abnormal sensitivity to clicks: if the abnormal vestibular activation were highly frequency selective, dependent on temporal summation at one or more synapses or an indirect consequence of reflexes evoked by the sound, ${ }^{6}$ then no abnormality would be expected in response to the very short acoustic stimulation caused by clicks. Conversely, abnormal vestibular sensitivity to clicks would strongly suggest an intrinsic abnormality of vestibular receptors, most probably the saccule, the vestibular receptor with the greatest sensitivity to this form of stimulus. ${ }^{9-12}$

In view of the potential importance of our initial finding we have investigated a series of patients with Tullio phenomenon using clicks as well as galvanic vestibular stimulation, the second to assess the excitability of vestibular pathways proximal to the receptors. We have in addition used the opportunity presented by these patients' low thresholds to click activation to demonstrate the pattern of short latency vestibular reflex projections to other neck and limb muscles.

\section{Methods}

Click evoked vestibulocollic reflexes were recorded from seven patients who had presented with symptoms suggestive of the Tullio phenomenon to the National Hospital for Nervous Diseases, Queen Square and from 25 normal volunteers. One of the patients has been 
Summary of clinical features and click responses for patients with the Tullio phenomenon

\begin{tabular}{|c|c|c|c|c|c|c|}
\hline Patient & Sex, age & $\begin{array}{l}\text { Symptomatic } \\
\text { ear }\end{array}$ & $\begin{array}{l}\text { Duration } \\
\text { (y) }\end{array}$ & $\begin{array}{l}\text { Sound threshold } \\
\text { frequency }(\mathrm{dB}(\mathrm{kHz}))\end{array}$ & $\begin{array}{l}p 13 / n 23 \\
\text { (a) } 75 d B\end{array}$ & $\begin{array}{l}\text { p13/n23 } \\
\text { threshold (dB) }\end{array}$ \\
\hline 1 & $\mathrm{M}, 44$ & $\mathrm{~L}$ & 8 & $110(0.5)$ & 1.17 & 65 \\
\hline 2 & $\mathrm{~F}, 28$ & $\mathrm{~L}$ & 11 & $105(1,2)$ & 0.86 & $<70$ \\
\hline 3 & $\mathrm{~F}, 22$ & $\mathrm{R}^{\star}$ & 5 & $100(1)$ & 1.04 & $<60$ \\
\hline 4 & $M, 48$ & $\mathrm{R}$ & 8 & $105(0.5)$ & 1.12 & $<60$ \\
\hline 5 & $M, 63$ & $\mathrm{~L}$ & 12 & $\dagger$ & 0.97 & $<75 \ddagger$ \\
\hline \multirow[t]{2}{*}{6} & $M, 57$ & $\mathrm{R}$ & 18 & $110 \$$ & $>1.139$ & $<60$ \\
\hline & & $\mathrm{L}^{\star}$ & 18 & $110 \$$ & 0.27 ฯ & $<80$ \\
\hline 7 & F, 56 & $\mathrm{~L}^{\star}$ & 10 & $110(0.5,1)$ & $\star \star$ & 77 \\
\hline
\end{tabular}

^Surgical exploration of the middle ear, which in all three cases was normal. tpatient developed oscillopsia with $90 \mathrm{~dB}$ stimuli.

$\ddagger$ Not tested with clicks of $<75 \mathrm{~dB}$ intensity.

$§$ Frequency not recorded.

TTested at $70 \mathrm{~dB}$ NHL, following attempted stapediopexy on the left.

$\star \star$ Responded in one of two runs to clicks of $73 \mathrm{~dB}$ intensity.

Sound threshold=intensities and frequencies required to produce eye movements; p13-n23@75 $\mathrm{dB}=$ the peak to peak amplitude of the p13-n23 potential expressed as a ratio of the mean background rectified EMG level; p13-n23 threshold=threshold for evoking these potentials in the ipsilateral sternocleidomastoid muscle.

sensation of tilting during audiometric assessment. A total of 25 normal volunteers, recruited from laboratory and hospital staff and their relatives, and who had no history of either neurological or vestibular abnormalities, were included to provide reference values. Their ages ranged from 22 to 65 years, eight were men and 17 were women.

Continuous tone intensities are measured in $\mathrm{dB}$ International Standards Organisation (ISO) and click intensities in $\mathrm{dB}$ NHL (average normal hearing level; $0 \mathrm{~dB}=45 \mathrm{~dB}$ sound pressure level (SPL)).

\section{CLINICAL FINDINGS}

Several characteristics of the seven patients are summarised in the table. One patient was affected bilaterally (patient 6), giving eight symptomatic ears (five left, three right). The duration of symptoms varied from 5 to 18 years (mean 10.3). All had symptoms of vestibular activation by sound either as sound induced oscillopsia or sound induced imbalance, or both. Two patients (Nos 1 and 2) had noted head jerks precipitated by sound. Several patients had additional symptoms: three complained of a pressure sensation or pain in the affected ear, three complained of hyperacusis selectively for bone conducted sounds (for example, hearing their eyes move, or the road through a bicycle seat) and three had tinnitus, in one case pulsatile. One patient had had a series of episodes of vertigo and mild tinnitus 22 years previously tentatively diagnosed as Ménière's disease (patient 5), one, affected bilaterally, was exposed repeatedly to loud sounds in his employment (patient 6), and one had a history of migraine (patient 3). Routine neurological examination showed no abnormalities, apart from a tendency for the Weber test to localise to the affected ear, when tested with a $128 \mathrm{~Hz}$ tuning fork. Hennebert's sign (eye movements induced by raising the pressure in the external auditory canal) was positive in two patients (Nos 2 and 3). The results of caloric testing were normal in three (Nos 1, 4,and 6). Three patients had mild canal pareses, ipsilateral to the affected ear (Nos 2, 3, and 7) and one patient (No 5) had a directional preponderance. Pure tone audiograms were usually normal, but showed low frequency hearing loss in three symptomatic ears
(Nos 2 and 6, bilaterally). Conventional cranial imaging with CT was normal in all patients, as was MRI in the four patients so examined. Three patients had their middle ears explored (by Mr H. Ludman and Mr G Brookes) but no abnormality was found in any case. Soundinduced eye movements were shown to be present in six patients and characterised in five. In these five patients the lowest threshold for inducing eye movements varied between 100 and $110 \mathrm{~dB}$, and the most effective frequency was either $500 \mathrm{~Hz}, 1 \mathrm{kHz}$, or $2 \mathrm{kHz}$ (table). The induced eye movements, measured during stimulation of five ears, consisted of torsional slow phases with movement of the upper pole directed away from the stimulated ear associated with, in two patients, upward deviation. Nystagmus, when present, beat in the opposite direction (see Bronstein et $a l^{13}$ for a detailed desciption of the responses of patient 7).

\section{CLICK EVOKED VESTIBULAR ACTIVATION}

Methods of click evoked vestibular activation were similar to those previously published. ${ }^{814}$ The patients were tested using a custom designed circuit which delivered $0.1 \mathrm{~ms}$ long square wave pulses to calibrated earphones (TDH 49: Telephonics Corp, New York, USA). All click intensities are expressed with respect to the typical normal hearing threshold for such clicks (taken as $45 \mathrm{~dB}$ SPL). With this reference, a $100 \mathrm{~dB}$ NHL click corresponds to a pulse with an amplitude of about $9 \mathrm{~V}$ delivered to the earphones. For technical reasons, the softest click that could be given to patient 5 was $75 \mathrm{~dB}$ NHL, but the other patients were tested down to $60 \mathrm{~dB}$ NHL. Data were collected using a 1401plus laboratory interface (Cambridge Electronic Design, Cambridge, UK) with commercial software (SIGAVG 6.13) which allowed conditional averaging. In all click studies, the stimuli were delivered pseudorandomly either to the right or left ear at 3/s using a sampling rate of $5 \mathrm{kHz}$ and with averages made of 128 or 256 trials. Surface EMG recordings were made using $9 \mathrm{~mm}$ silversilver chloride electrodes (SLE, London, UK). For studies of the sternocleidomastoid muscles, active electrodes were placed over the muscle belly on both sides, $6-8 \mathrm{~cm}$ above its sternal insertion with reference electrodes placed over the sternoclavicular joints. To produce tonic activation of both sternocleidomastoid muscles, recordings were made with the patients lying, and lifting their heads off the examination bed. The 25 normal volunteers were recorded while sitting and activating their neck muscles by pressing their foreheads against a restraint. The normal volunteers were studied using clicks generated by an ST10 Evoked Potential Stimulator (Medelec, Surrey, UK). For both the patients and the normal volunteers, the first intensity tested was usually $95 \mathrm{~dB}$ (NHL). Thereafter the effects of $100 \mathrm{~dB}$ and $90 \mathrm{~dB}$ clicks were tested. If $\mathrm{p} 13-\mathrm{n} 23$ responses were evident with the $90 \mathrm{~dB}$ clicks the normal volunteers were retested with clicks progressively less intense, using $5 \mathrm{~dB}$ decrements, until the p13-n23 response was lost. The first intensity for which a p13-n23 response was evident for stimulation of the ipsilateral ear was defined as 
the threshold. Testing of the patients was similar but because they had large responses, the click was usually decremented in $10 \mathrm{~dB}$ steps. The peak to peak amplitude of the p13-n23 response was measured and then expressed as a ratio of the background mean rectified EMG level (baseline EMG) to correct for the effects of differences in the tonic level of muscle activation as well as to ensure that adequate levels of muscle activation were achieved. ${ }^{14}{ }^{15}$ For studies of posterior neck muscles, three active recording electrodes were placed just below the occiput, one in the midline and the two others $5 \mathrm{~cm}$ to either side of it. A common reference was used, placed over the C7 spine. For both the anterior and posterior neck recordings, a negative potential at the active electrode caused an upward deflection. We examined the averaged responses during right and left ear stimulation using $95 \mathrm{~dB}$ clicks under three different conditions: isometric rotation to the right, isometric rotation to the left, and isometric neck extension. These manoeuvres were designed to activate predominantly the right or left splenius muscles (rotation right and left, respectively) or the neck extensors while isometric contractions minimised electrode movement with respect to the underlying muscles. Mean rectified EMG levels were used to monitor the levels of muscle activation.

For studies of the effect of clicks on leg muscles, we recorded EMG activity from the tibialis anterior with one electrode placed over the muscle belly and a reference electrode on the fibular head. In one set of experiments, subjects stood erect leaning slightly backwards to produce some activity in the anterior tibial muscles. In another set of tests, the subjects sat down and dorsiflexed their ankles to produce about the same amount of activity in the tibialis anterior. In both tests, clicks were given randomly to each ear while subjects rotated their head as far as possible to the left or right in separate blocks of trials. Responses were measured from the average rectified EMG.

GALVANIC VESTIBULAR STIMULATION

Postural responses to galvanic vestibular stimulation were elicited in five of the seven patients (patients 3 and 5 not studied). The methods used were similar to those previously published. ${ }^{16}$ Galvanic stimuli were applied via two 3 $\mathrm{cm}$ diameter electrodes made of soft dental metal which were fixed to the mastoid processes with collodion glue and filled with conductive electrode jelly. A custom designed constant current stimulator was used to pass a current of 0.5 $\mathrm{mA}$ between the electrodes for a duration of 4 seconds. An infrared emitting diode was fixed to the neck of the subject over the prominence of the spinous process of the seventh cervical vertebra and its position measured using a motion analysis system (Selspot II, Selcom AB, Sweden).

Subjects stood on a fixed force plate (type 9281B, Kistler Instrumente AG, Switzerland) with their feet together, eyes closed, and head in the midposition. At a random time after eye closure (2-5 seconds) a galvanic stimulus was applied for 4 seconds. Data were collected for a period of 5 seconds starting 1 second before the stimulus was applied. At the end of a trial subjects opened their eyes and stood relaxed. The polarity of the stimulus (anode right, anode left) was varied in a pseudorandom manner across trials. Between 10 and 16 trials were collected for each of the two conditions and the results averaged.

Baseline stability of each subject standing with eyes closed was assessed from the mean speed of body motion (irrespective of direction) over the 0.25 second period before stimulation. The postural response to stimulation was measured in three ways: the mean speed, mean velocities in the frontal and sagittal planes, and the direction of induced body sway over the period 0.75 to 1.0 seconds after stimulus onset. The patients' results were compared with those obtained from a group $(n=19)$ of neurologically normal subjects.

\section{Results}

CLICK EVOKED RESPONSES IN SYMPTOMATIC PATIENTS WITH THE TULLIO PHENOMENON: STERNOCLEIDOMASTOID RECORDINGS Figure 1 shows the typical pattern that we recorded in patients with the Tullio phenomenon. The traces show EMG responses from the ipsilateral sternocleidomastoid muscle after clicks to the left or right ear at intensities from $60 \mathrm{~dB}$ to $100 \mathrm{~dB}$. The vestibulocollic reflex is the first positive/negative wave (positive is downwards) after the stimulus and is termed the p13-n23, corresponding to the usual latency of the peaks. In this patient, clicks to the left (normal) ear produced a p13-n23 response that had a threshold of around $90 \mathrm{~dB}$, whereas clicks to the right (clinically symptomatic) ear produced clear responses at much lower intensities $(<60 \mathrm{~dB})$.

In general, the EMG responses from the asymptomatic ears were similar to those in the 25 normal subjects (fig 1). The threshold ranged from $75 \mathrm{~dB}$ (in two subjects) to $100 \mathrm{~dB}$ (one subject), with the mean value being 86 $\mathrm{dB}$. The shaded area in fig 2 indicates the range of normal behaviour. In this figure, the size of the response has been expressed as a fraction of the background EMG activity, and is plotted against the intensity of the click.

The threshold of the p13-n23 response was usually lower in the patients' symptomatic ears than in any of the normal subjects. The sizes of the responses tested near threshold are shown in fig 2 and details are given in the table. In five of the seven patients, threshold was $<70 \mathrm{~dB}$, lower than in any normal subject. In patient 5 the softest click that could be employed at the time of testing was $75 \mathrm{~dB}$, but this gave a very clear response with an amplitude that was outside the range of normal values. Only patient 7 showed any overlap with the normal range. She had a mean threshold of $77 \mathrm{~dB}$, although in one of two repeat runs, a response was evident at 73 dB. Patient 6 had originally presented with bilateral symptoms of the Tullio phenomenon. However, he was tested with clicks after an attempted stapediopexy on the left, and the threshold on that side fell within the normal range. In the remaining six patients, responses 
from the asymptomatic ear all fell within the normal range, with thresholds ranging from 80 $\mathrm{dB}$ to $100 \mathrm{~dB}$.

Apart from their low threshold, responses from the affected side behaved in a manner very similar to that seen in normal subjects. The morphology of the response and the slope of the relation between intensity and size (fig 2 B) were the same as in normal subjects. The amplitude of the response seemed to saturate at high intensities of stimulation in patients, but this was also seen in some normal subjects who had large responses. Five of the patients volunteered that they developed oscillopsia in response to the click testing, with subjective tilt of vertical objects and thresholds between 85 and $100 \mathrm{~dB}$ (NHL). No normal volunteer noted such symptoms.
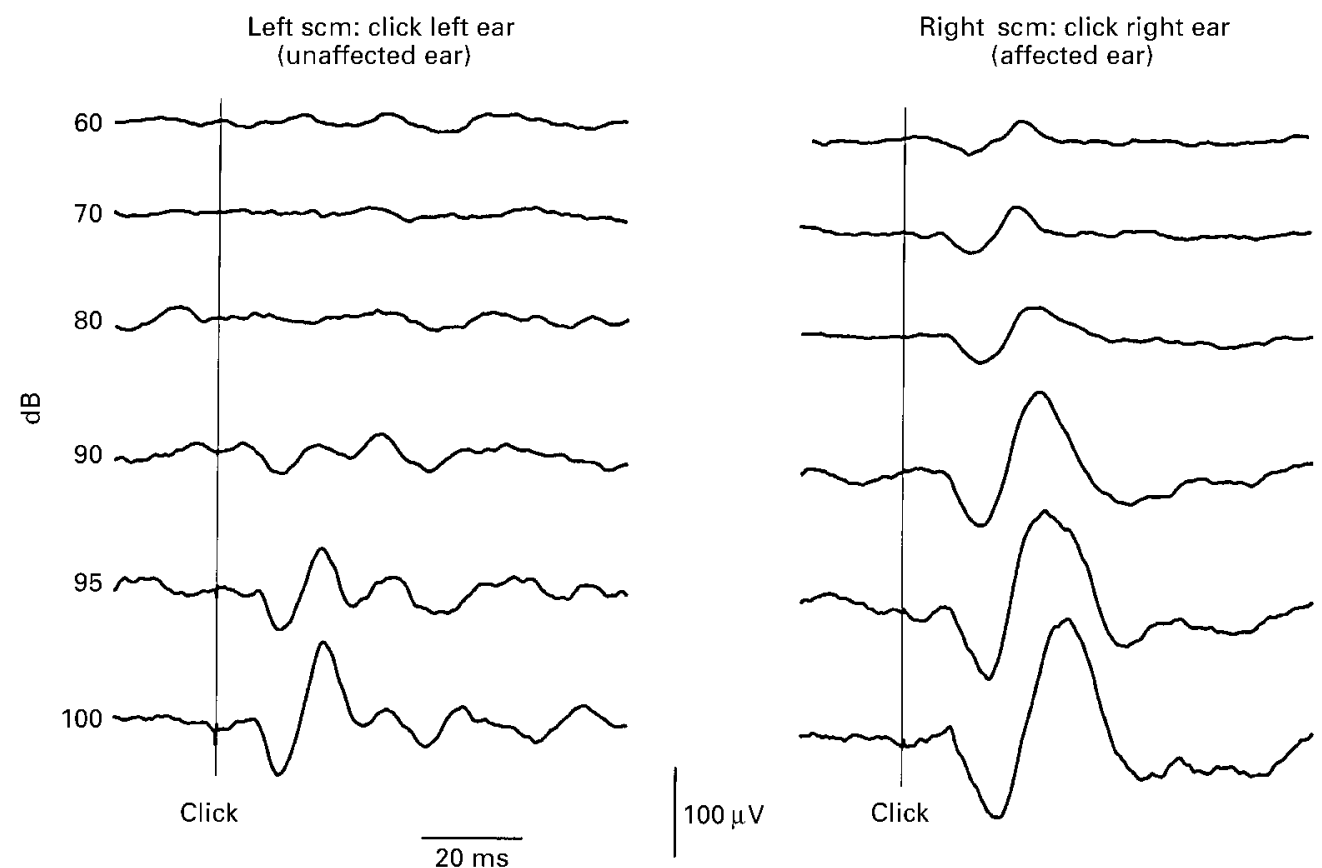

Figure 1 Patient 3, right Tullio phenomenon. Effects of clicks of different intensities given to each ear. The clicks were presented $20 \mathrm{~ms}$ after the start of the traces and means are shown of recordings made over the right and left sternocleidomastoid muscles (scm) in response to clicks given to the ipsilateral ear. The lowest intensity clicks used (60 dB) when given to the right ear evoked a p13-n23 response in the right scm, but the same intensity clicks given to the unaffected (left) ear had no recordable effect on the left scm. With increasing intensity, the response on the right side became progressively larger, such that, with the most intense clicks, shown on the bottom row, the p13-n23 amplitude increased to over $100 \mu \mathrm{V}, 2.9$ times the mean background EMG. Clicks given to the left ear evoked p13-n23 responses in the L scm with a threshold of $90 \mathrm{~dB}$, which was within the range of normal controls.
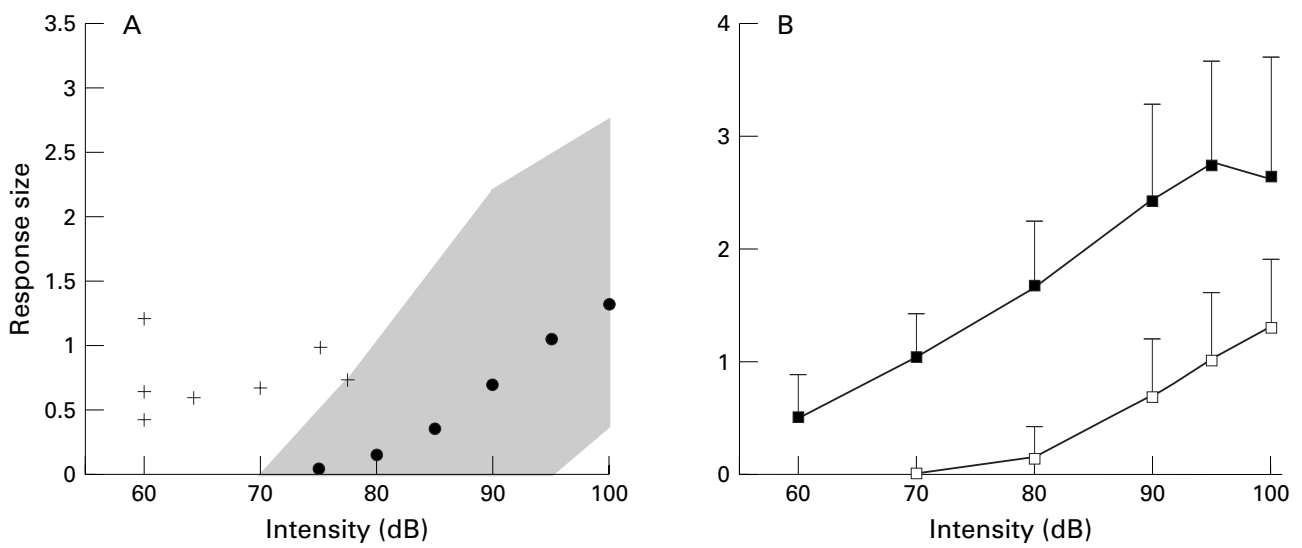

Figure 2 (A) Summary of the range of values recorded from the 25 normal volunteers in response to clicks and the responses obtained with the patients with the Tullio phenomenon with weak stimuli. All amplitudes have been expressed as a ratio of the mean prestimulus rectified EMG level to correct for differences in the level of tonic activation.$^{14}$ The shaded area shows the range of amplitudes seen for the ears of the 25 normal volunteers with click intensities varying between 70 and $100 \mathrm{~dB}$ NHL and the filled dots give the average values. The crosses show the size of the responses obtained in seven symptomatic ears of the patients with the Tullio phenomenon to weak click stimulation (the left ear of patient 6, which had a conductive hearing loss at the time of testing, is not shown). All the patients had very low thresholds for click activation of the vestibular apparatus and all but one (patient 7) lay outside the normal range. (B) shows the response behaviour for the patients' affected ears with stronger stimulation ( $n=6$ : patient 5 not tested, patient 6 right ear only). The mean (SD) (filled squares and bar) are shown as well as the corresponding values for the normal volunteers (open squares). The patients' responses seem to have been shifted to the left to become about $20 \mathrm{~dB}$ more sensitive than normal but the increase in response amplitude with increasing stimulus intensity above threshold is similar to that for normal controls. 
The results so far have been concerned only with the EMG response in the ipsilateral sternocleidomastoid muscle. This is because in normal subjects, it is unusual to see any early activity in the contralateral muscle. This was not the case in the patients. The highest intensity of stimulation $(100 \mathrm{~dB})$ of the symptomatic ear(s) always produced a short latency response of opposite polarity (negative-positive wave) in the contralateral sternocleidomastoid (fig 3). The threshold for its appearance was higher than that for the ipsilateral response, and varied from $80 \mathrm{~dB}$ to $100 \mathrm{~dB}$.
CLICK EVOKED RESPONSES IN SYMPTOMATIC PATIENTS WITH THE TULLIO PHENOMENON: POSTERIOR NECK AND LEG MUSCLES

The large size and low threshold of the click responses in patients encouraged us to explore the distribution of reflex responses in more detail. We began with the posterior neck muscles, the site where Bickford et $a l^{17}$ originally described the presence of click evoked myogenic potentials.

Five patients were studied using a click intensity of $95 \mathrm{~dB}$ and while turning the head against resistance to the left or the right to acti-
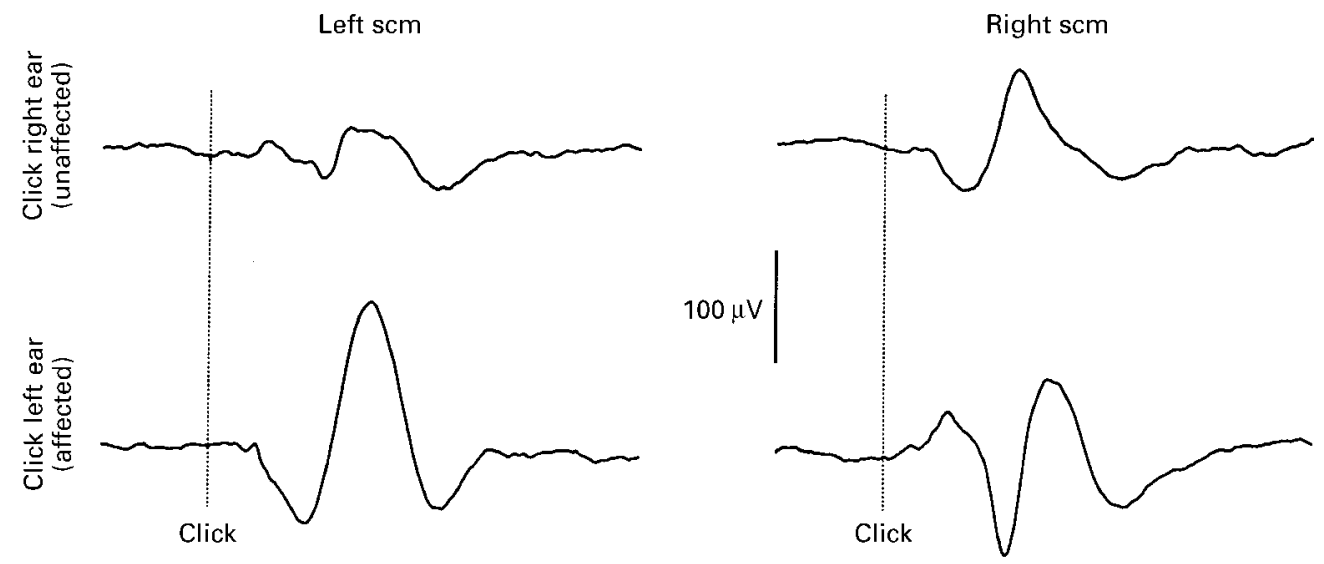

$20 \mathrm{~ms}$

Figure 3 Patient 1, illustrating the effects recorded from the contralateral and ipsilateral sternocleidomastoid muscles (scms) in response to stimulation of the affected ear (left) and the unaffected ear (right) at high intensities ( $95 \mathrm{~dB}$ clicks). With stimulation of the right ear (upper traces) there is a normal p13-n23 response in the ipsilateral scm and a small response contralaterally. With stimulation of the affected ear with same intensity clicks (lower traces) there is a large p13-n23 response present in the ipsilateral scm, but also a well formed crossed response recorded from the right scm. The latency of onset of the crossed response is similar to the ipsilateral one, but begins with a negativity.

Activate muscles by turning head

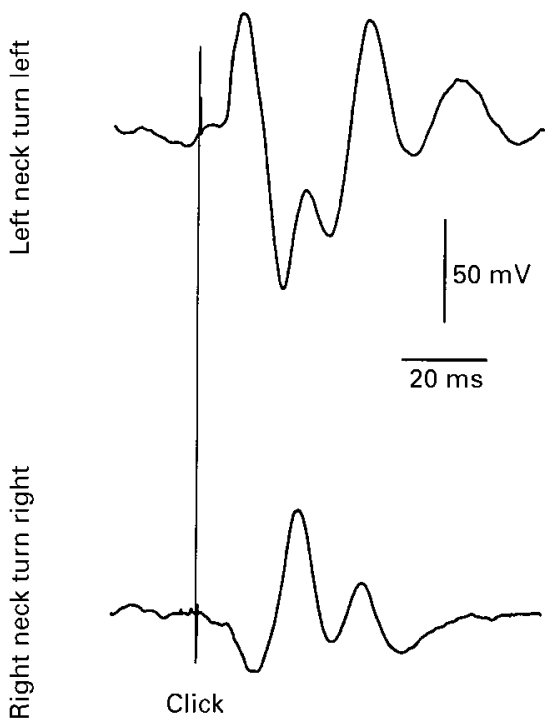

Activate muscles by extending neck

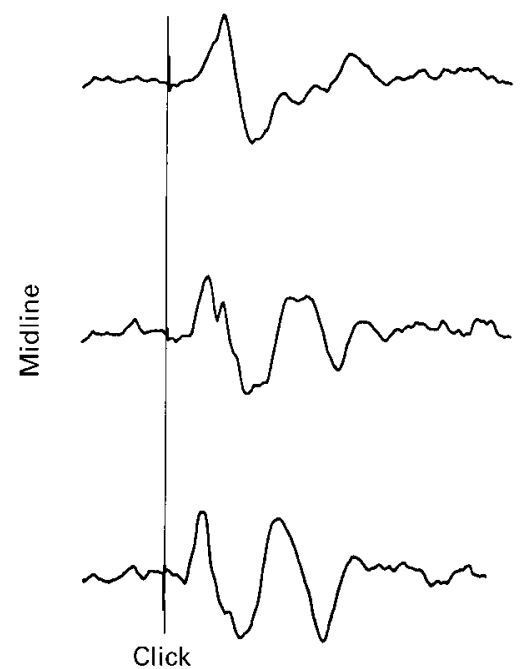

Figure 4 Patient 2, showing effects of a $95 \mathrm{~dB}$ click given to the left (symptomatic) ear in all cases. Recordings made from electrodes over the midline of the upper posterior neck ("inion" electrode) and $5 \mathrm{~cm}$ to the left and right of it. The left two traces show the effects of stimulating the left ear during rotation to the left (left neck electrode, upper left) and during rotation to the right (right neck electrode, lower left). The initial potential is negative (upgoing) in the ipsilateral neck muscles and positive in the contralateral ones. The right half of the figure shows recordings made simultaneously from all three electrodes during isometric extension of the neck. Under these conditions stimulation of the left ear induces similar potentials in all three electrodes, beginning with an initial negativity. Clicks given $20 \mathrm{~ms}$ after the start of the traces in all cases. 


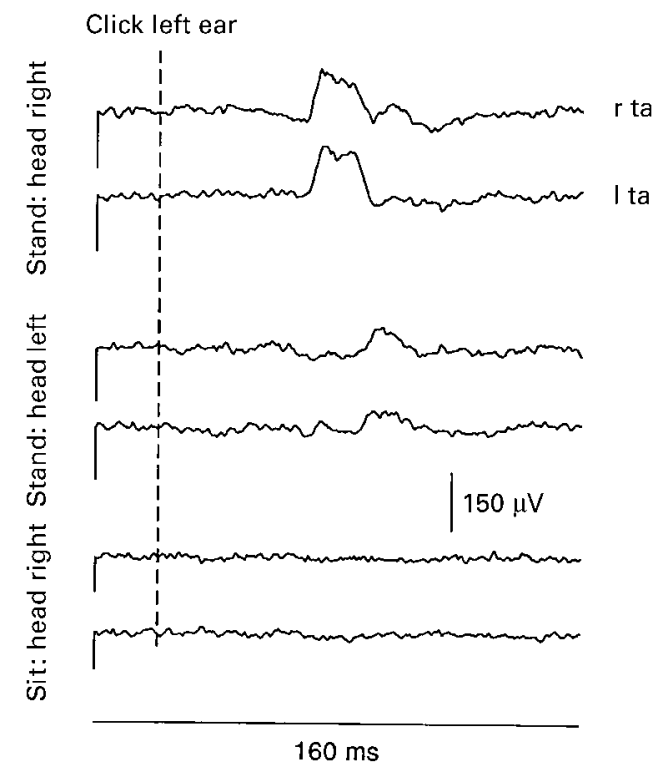

Figure 5 Patient 2, responses to clicks (95 dB) given to the symptomatic (left) ear and recorded from both tibialis. anterior muscles, during stance and when seated. Rectified averages are shown $(n=256$ sweeps). With the head turned to the right (uppermost traces), there is an increase in activity (excitation) of both muscles, beginning at $50 \mathrm{~ms}$ and lasting $20 \mathrm{~ms}$. The effect reverses with rotation of the head to the left (middle traces). When seated, despite the subject maintaining similar levels of tonic muscle activation to that when standing (level of mean rectified activity shown by height of initial line), there was no effect after the click.

vate the posterior rotators of the head (mainly splenius). Short latency responses could always be obtained, which usually had the same polarity as those evoked in the contralateral sternocleidomastoid. Thus, consider a click given to the right ear during rotation of the head to the left with the right sternocleidomastoid and the left splenius muscles active. The click evokes a positive/negative response in both muscles with about the same latency. A positive/negative response is inhibitory, ${ }^{18}$ so that in functional terms, a right ear click inhibits rotation of the head to the left. If the click is to the left ear, then the polarity of the response is inverted (fig 4). Click stimulation during neck extension usually evoked an initial negativity in all three electrodes with a mean latency of $11.5 \mathrm{~ms}$ and additional potentials were present for up to 50 ms after the stimulus. The potentials evoked from stimulating the asymptomatic ear were more variable but often followed the same pattern as recorded from the symptomatic ear.

We also investigated whether click stimuli could evoke responses in leg muscles which have been previously shown to receive short latency vestibular projections. ${ }^{19}{ }^{20}$ Responses to $95 \mathrm{~dB}$ clicks were present in all patients, and as the responses described after galvanic stimulation, they depended on the direction in which the head was turned, and whether the patient stood or was seated. As is customary for recording these reflexes, we measured the leg responses using averaged records of rectified rather than unrectified EMG. As a consequence, the typical biphasic EMG potential was replaced by periods of increased or decreased activity. Figure 5 illustrates the typical pattern produced by clicks to the symptomatic (left) ear in patient 2 . When the subject stood with the head turned to the right (left ear forwards), clicks to the left ear evoked an increase in activity of tibialis anterior with a
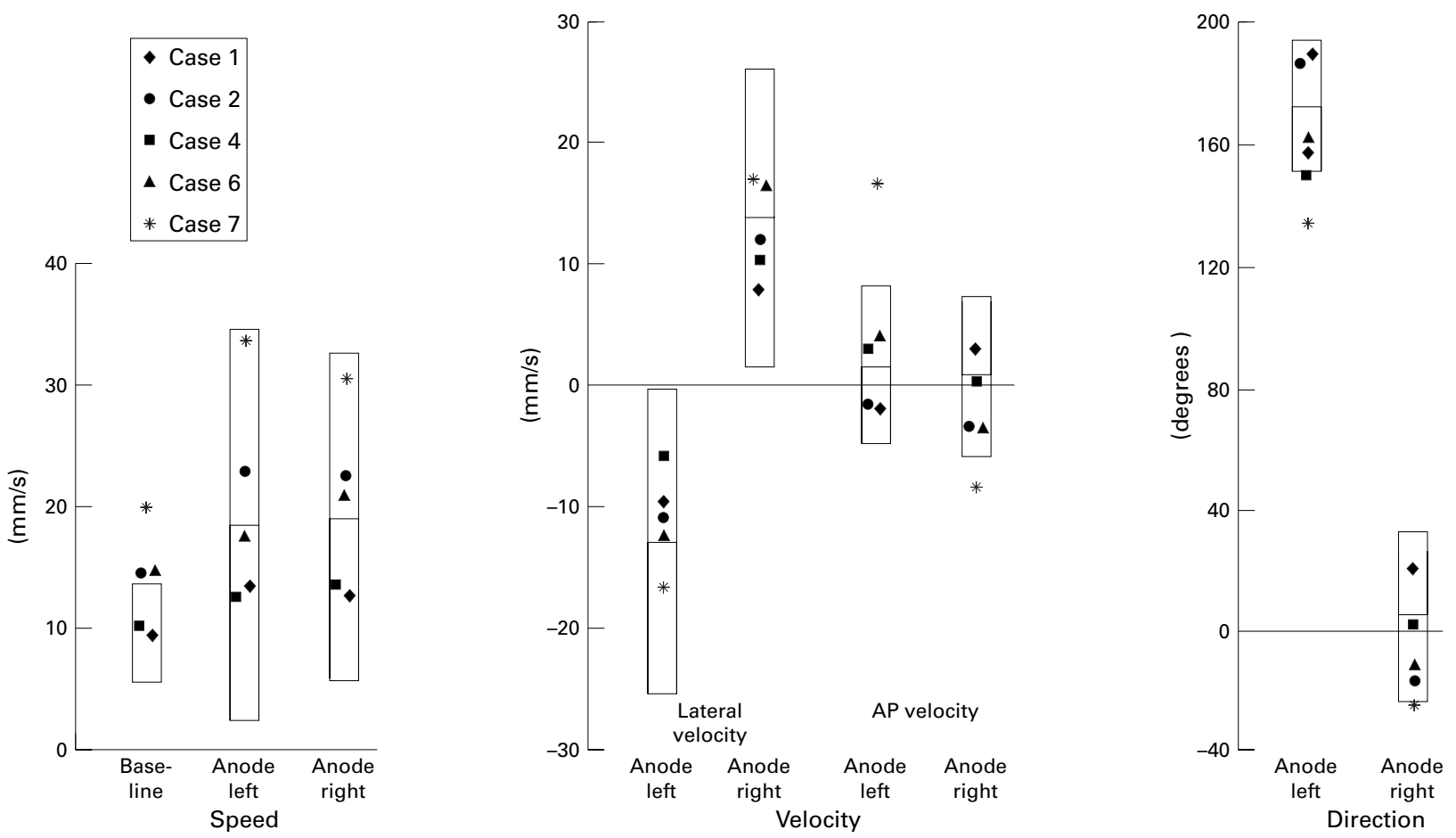

Figure 6 Summary of galvanic induced sway in five of the patients, studied in a darkened room with their eyes shut. The boxes indicate the mean (2SD) of normal responses. The left part of the diagram, "Speed", shows the sway speed, irrespective of direction, before stimulation, and the effects of galvanic stimulation. In the middle part of the figure ("Velocity") the sway has been resolved into components in the anteroposterior ("AP") and lateral planes. The final part of the figure ("Direction") indicates the direction of the induced movement. Patient 7 had the largest responses to stimulation, but also had a greater spontaneous sway than normal. The remainder of the patients had responses to galvanic stimulation which were within the normal range, irrespective of the side stimulated. 
latency of about $50 \mathrm{~ms}$, and a duration of $20 \mathrm{~ms}$. There was a small reduction in rectified EMG at this latency when the head was turned to the left (left ear backwards) followed by a later small increase with an onset latency of $75 \mathrm{~ms}$. When seated, clicks had no effect on the EMG activity.

The mean data from all seven subjects showed that clicks to the affected ear evoked responses in the tibialis anterior which had an average latency of 52 (SEM 1) ms and a duration of 21.4 (SEM 2) ms. The size of responses was equal in both legs, so that results from each were combined. The area of the response was expressed as a percentage of the background level of EMG activity before stimulation. When the head was turned so that the stimulated ear was anterior, responses averaged 131 (SEM 6) $\%$ of background, whereas if the head was turned in the opposite direction the responses were only 95 (SEM 6)\% of background. There was no discernible response when sitting.

Clicks to the non-symptomatic ear had less effect on leg muscle activity. In the six patients in whom only one ear was symptomatic (omitting patient 6 ), the mean response size evoked by stimulating the symptomatic ear (with the head rotated to bring it anterior) was $128 \%$ of the background EMG, whereas it was only $112 \%$ of the background when the asymptomatic ear was stimulated ( $\mathrm{p}<0.05$, paired $t$ test).

PATIENTS WITH THE TULLIO PHENOMENON:

GALVANIC VESTIBULAR STIMULATION

In normal subjects, galvanic stimulation produces consistent sway of the body in a direction roughly towards the ear on which the anode is fixed. ${ }^{21}$ Body motion at the level of $\mathrm{C} 7$ reaches a maximum speed 0.75 to 1 second after the stimulus onset. ${ }^{16}$ Figure 6 illustrates the mean (2 SD) values of speed, velocity, and direction over this epoch for the normal group.

Three of the five patients (patients 2,6, and 7 ) were more unstable than normal when they stood with eyes closed and feet together (fig 6, baseline speed). However, the size and direction of galvanically induced postural responses were within normal limits for four of the five patients. In one patient (No 7) the size of the response was normal but the direction of induced body motion was abnormal.

\section{Discussion}

Patients with the Tullio phenomenon show hypersensitivity of the vestibular system to sound, and report symptoms of oscillopsia and unsteadiness to sounds of moderate intensities such as the ringing of a telephone. The present report shows that such patients are characterised by an abnormally low threshold for vestibular activation by impulse noise (clicks), a specific abnormality given that responses to other forms of vestibular activation, such as caloric or galvanic stimulation, were not increased. Further experience with click evoked vestibular testing will be required to determine its diagnostic value in the Tullio phenomenon compared with methods based on eye movement recordings.
RESPONSES TO CLICK STIMULI

Click stimuli are often used in conjunction with EEG recordings to test the integrity of brainstem auditory pathways. However, in 1964, Bickford et $a l^{17}$ noted that loud clicks could also produce short latency reflex EMG changes (myogenic responses) in posterior neck muscles (the inion response), and it is this type of response which has been studied here. A recording site over the sternocleidomastoid muscles was used because the midline recording site chosen by Bickford et al can mix potentially opposing input from both sides of the neck, and thereby confuse the results. With the sternocleidomastoid recording site, the response to clicks is seen in unrectified averages of surface EMG and consists of an initial positivenegative deflection with onset latency of approximately $8 \mathrm{~ms}$, followed by a more variable response beginning $30 \mathrm{~ms}-40 \mathrm{~ms}$ after the click. With unilateral clicks, the initial response is probably generated by a three neuron arc and consists of the $\mathrm{p} 13-\mathrm{n} 23$ potential. This potential occurs ipsilaterally to the stimulus whereas later, symmetric responses which are not vestibular dependent occur bilaterally. ${ }^{14}$

In normal subjects, the lowest threshold for evoking click responses was $75 \mathrm{~dB}$ (NHL), and click intensities of $85 \mathrm{~dB}$ (NHL) or higher were required for most normal subjects to show responses. All the patients had thresholds which were less than the average threshold for normal subjects ( $86 \mathrm{~dB}$ ), and some of the patients were nearly $30 \mathrm{~dB}$ more sensitive than this. Only one patient (No 7) had a threshold which overlapped with those of the most sensitive normal subjects, indicating that click thresholds are likely to be a useful diagnostic test for this condition. As noted in the Introduction there is now strong evidence that the early part of the myogenic click response is produced by stimulation of vestibular receptors, most likely originating in the saccule. If so, this implies that otolith receptors in many (if not all) patients currently presenting with Tullio phenomena are hypersensitive to sound. Furthermore, the short latency of this reflex implies that the increased sensitivity of these patients to clicks cannot be a consequence of displacement of the stapes induced by the stapedial reflex (cf Dieterich et $a l^{6}$ ). Thus the patients with the Tullio phenomenon seem to be characterised by excessive transmission of sound energy to the saccule. The form of the potential and its behaviour at suprathreshold intensities were the same in patients as in normal subjects, implying that the central neural circuitry involved in the click potential functions normally in patients.

We propose that the Tullio phenomenon may be defined either in terms of the response to continuous tones (sound induced eye movements $<115 \mathrm{~dB}$ SPL or $110 \mathrm{~dB}$ ISO), or in terms of the response to short duration clicks (click evoked myogenic response thresholds $\leqslant$ intensities of $115 \mathrm{~dB}$ SPL (=70 dB NHL)). 
DISTRIBUTION OF CLICK EVOKED MYOGENIC POTENTIALS

We were able to use the opportunity presented by these patients' vestibular sensitivity to clicks to explore short latency reflex projections to neck and leg muscles. The first includes useful clarification of the inion response originally described by Bickford et $a l,{ }^{17}$ whereas the second gives insight into possible vestibulospinal projections in humans.

In addition to the ipsilateral p13/n23 response, an equally early response of opposite polarity was recorded from the contralateral sternocleidomastoid with stimulation of the patients' symptomatic ears. These crossed responses are present in some normal subjects (unpublished data) but were not reported in the initial description of click evoked vestibulocollic reflexes, ${ }^{14}$ probably due to their higher threshold compared with the ipsilateral reflex effects. We interpret these findings as indicating that there is actually a bilateral, three neuron projection from each vestibular apparatus to the two sternocleidomastoid muscles, with reciprocal actions. The ipsilateral effect has the lower threshold and is inhibitory whereas the contralateral effect is excitatory.

In the posterior neck, short latency reflex responses to clicks were best seen in recording sites to the right and left of the midline over the splenius muscles. As with responses in the sternocleidomastoid, we think that the earliest potentials were caused by sound activation of vestibular receptors. Support for a vestibular origin includes the larger responses present during stimulation of the affected ear in patients with the Tullio phenomenon and the reversal in sign depending on the side being stimulated, features unlike cochlear dependent responses. ${ }^{14}$ Recordings of the inion response described by Bickford et $a l^{17}$ did not show an effect of side, presumably because their technique mainly activated neck extensors, which, unlike neck rotators, do not show lateralised effects.

As noted in the results, the pattern of the response to clicks in the neck was to produce an initial positive/negative potential in the ipsilateral sternocleidomastoid muscle and contralateral splenius. Normally, these two muscles act as synergists and rotate the head away from the stimulated ear. Given that an initial positivity in the average unrectified EMG seems to correlate with inhibition of motor unit activity, ${ }^{18}$ this means that a click inhibits rotation of the head away from the stimulated ear. The fact that potentials of the opposite polarity could be seen (at higher intensities of stimulation) in the antagonist muscles suggests that the reflex might also facilitate rotation of the head towards the side of stimulation. These reflex effects are likely to be the mechanism of the head jerks present in patients 1 and 2 .

We also explored click evoked reflexes in leg muscles. Our evidence that these are vestibular dependent is indirect, but compelling. The responses were larger from the affected ear, occurred at similar latencies to those described after galvanic vestibular stimulation and, similar to galvanic stimulation, the type and presence of a response was dependent on the posture of the subject. ${ }^{21}$ The latency for EMG changes in the tibialis anterior was close to that reported by Dieterich et $a l .{ }^{6}$ Click evoked responses were only seen in leg muscles if the subjects were standing, and their polarity was altered by head rotation. Similar effects on leg muscles can be recorded after clicks in some normal subjects, ${ }^{22}$ suggesting that the responses seen for the patients are generated through normal central reflex pathways.

\section{GALVANIC SWAY RESPONSES}

In standing subjects, application of a small DC (galvanic) current across the mastoid processes makes the body sway towards the side of the stimulating anode. ${ }^{23}$ Remarkably, the direction of sway changes with rotation of the head or trunk. ${ }^{21}$ Thus, an anode on the right mastoid produces lateral sway to the right if subjects stand with their heads facing forwards, whereas if the head is turned to the left, sway will be in the anterior direction. The induced sway is thought to be produced through activation of vestibulospinal and perhaps other descending pathways. ${ }^{19}$

The responses to galvanic stimulation were within normal limits in four of the five patients tested. In the fifth patient, there was evidence only of an abnormal directional bias but no hypersensitivity. As the site of action of galvanic stimulation lies proximal to the probable site of action of the clicks (the hair cells ${ }^{9}$ ), these findings are consistent with our earlier conclusions that, in most patients, the central pathways mediating vestibular induced effects on the legs are normal, and that the Tullio phenomenon is caused by hypersensitivity of the vestibular end organs themselves. The directional bias in the fifth patient may have been part of an adaptive response to her disorder.

IMPLICATIONS FOR POSSIBLE MECHANISMS OF THE TULLIO PHENOMENON

Animal experiments have shown that, of all components of the vestibular system, it is the saccule that is most readily activated by normally transmitted sound. ${ }^{9-11}$ It is therefore likely that this structure mediates the myogenic responses to clicks which can be recorded in normal subjects. Because click responses in patients with the Tullio phenomenon are similar to those in normal subjects in all respects except threshold, it seems likely that hypersensitivity of the saccule to sound energy is a common feature of the condition. Whether or not the patients' clinical symptoms are the result of saccular stimulation is unclear. Most of them did report symptoms of oscillopsia during click testing, and these may have been caused by activity in saccular projections to eye muscles. ${ }^{24}{ }^{25}$ However, it is possible that other vestibular afferents may also have been stimulated, and that subsets of patients, depending perhaps on the aetiology of their disorder, have different proportions of additional canal or utricular sensitivity. ${ }^{6}$

We propose that click evoked vestibular activation has an important role in the investigation and diagnosis of patients with suspected Tullio phenomenon. Vestibular activation by clicks with intensities of $70 \mathrm{~dB}$ (NHL) and 
below does not occur in normal subjects and seems to be indicative of the presence of the Tullio phenomenon. Finally, experimental evidence that click activation is selective for saccular afferents suggests that saccular activation contributes to the sound induced vestibular symptoms which are characteristic of the Tullio phenomenon.

JGC held a Welcome/Ramaciotti Foundations Travel Gran while undertaking these studies in London and his research was supported by the National Health and Medical Research Council of Australia. Christine Urban kindly translated the work of Békésy. ${ }^{1}$

1 Békésy $\mathrm{G}$ von. Uber akustische Reizung des Vestibularapparates. Pflügers Archiv Gesamte Physiol Menschen Tiere rates. Pflügers Arch

2 Dickson EDD, Chadwick DL. Observations on disturbances of equilibrium and other symptoms induced by jetengine noise. F Laryngol Otol 1951;65:154-65.

3 Parker DE, Tubbs RL, Littlefield VM. Visual-field displacements in human beings evoked by acoustical transients. $\mathscr{f}$ Acoust Soc Am 1978;63:1912-18.

4 Cawthorne T. The contributions of surgery to the problems of neuro-otology. Br Med Bull 1956;12:143-5.

5 Kacker SK, Hinchcliffe R. Unusual Tullio phenomena. $\mathcal{F}$ Laryngol Otol 1970;84:155-66.

6 Dieterich M, Brandt Th, Fries W. Otolith function in man: results from a case of otolith Tullio phenomenon. Brain 1989;112:1377-92.

7 Minor LB, Solomon D, Zinreich JS, et al. Sound or pressure induced vertigo due to bone dehiscence of the superior induced vertigo due to bone dehiscence of the superior
semicircular canal. Arch Otolaryngol Head Neck Surg 1998; 124:249-58.

8 Colebatch JG, Rothwell JC, Bronstein A, et al. Click-evoked vestibular activation in the Tullio phenomenon. $\mathcal{F}$ Neurol Neurosurg Psychiatry 1994;57:1538-40.

9 Young ED, Fernández C, Goldberg JM. Responses of squirrel monkey vestibular neurons to audio-frequency sound and head vibration. Acta Otolaryngol (Stock) 1977;84:352-60.
10 Didier A, Cazals Y. Acoustic responses recorded from the saccular bundle on the eighth nerve of the guinea pig. saccular bundle on the eig
Hearing Res $1989 ; 37: 123-8$.

11 McCue MP, Guinan JJ. Acoustically responsive fibers in the vestibular nerve of the cat. $\mathcal{F}$ Neurosci $1994 ; 14: 6058-70$

12 Murofushi T, Curthoys IS, Topple AN, et al. Responses of guinea pig primary vestibular neurons to clicks. Exp Brain Res 1995;103:174-8.

13 Bronstein AM, Faldon M, Rothwell J, et al. Clinical and electrophysiological findings in the Tullio phenomenon. Acta Otolaryngol Suppl (Stock) 1995;520:209-11.

14 Colebatch JG, Halmagyi GM, Skuse NF. Myogenic potentials genrated by a click-evoked vestibulocollic reflex. 7 Neurol Neurosurg Psychiatry 1994;57:190-7.

$15 \mathrm{Lim}$ CL, Clouston P, Sheean G, et al. The influence of voluntary EMG activity and click intensity on the vestibular click evoked myogenic potential. Muscle Nerve 1995;18: 1210-13.

16 Pastor MA, Day BL, Marsden CD. Vestibular induced postural responses in Parkinson's disease. Brain 1993;116: 1177-90.

17 Bickford RG, Jacobson JL, Cody DTR. Nature of averaged voked potentials to sound and other stimuli in man. Ann N Y Acad Sci 1964;112:204-23.

18 Colebatch JG, Rothwell JC. Vestibular-evoked EMG responses in human neck muscles. F Physiol (Lond) 1993;473:18P.

19 Britton TC, Day BL, Brown P, et al. Postural electromyographic responses in the arm and leg following galvanic vestibular stimulation in man. Exp Brain Res 1993;94:14351.

20 Fitzpatrick R, Burke D, Gandevia SC. Task-dependent reflex responses and movement illusions evoked by galvanic vestibular stimulation in standing humans. F Physiol (Lond) 1994;478;363-72.

21 Nashner LM, Wolfson P. Influence of head position and proprioceptive clues on short latency postural reflexes evoked by galvanic stimulation of the human labyrinth. Brain Res 1974;67:255-68.

22 Watson SRD, Colebatch JG. Vestibular-evoked EMG responses in soleus: a comparison between click and responses in soleus: a comparison between click and
galvanic stimulation. Exp Brain Res 1998;19:504-10.

23 Coats AC. Effect of different stimulus parameters on the Coats AC. Effect of different stimulus parameters on the
galvanic body-sway response. Ann Otol 1973;82:96-102.

24 Fluur E, Mellström A. Saccular stimulation and oculomoto reactions. Laryngoscope 1970;80:1713-21.

25 Curthoys I. Eye movements produced by utricular and saccular stimulation. Aviat Space Environ Med 1987;58(suppl 9):A192-7.

Continued from page 669

native DNA of $86 \mathrm{IU} / \mathrm{ml}$ (normal range $<40 \mathrm{IU} / \mathrm{ml}$ ) and an antiparietal cell antibody titre of 21 $\mathrm{U} / \mathrm{ml}$ (normal range $<10 \mathrm{U} / \mathrm{ml}$ ). The $\mathrm{HBsAg}$ was positive with $128 \mathrm{IU} / \mathrm{ml}$ (normal range $<1$ $\mathrm{IU} / \mathrm{ml}$ ). All other tests, including antinuclear antibodies, were unremarkable. The patient was managed conservatively. There was a gradual improvement in her neurological deficits. Four months later she still complained of double vision, but refused further diagnostic or therapeutical interventions.

Cerebral artery aneurysms usually develop at bifurcating branches of the arteries of the circle of Willis. Perforating artery aneurysms have been described only in occasional patients. There were no findings suggesting a "mycotic" aneurysm due to an infectious aetiology in our case. Before the advent of contemporary diagnostic and therapeutic techniques "microaneurysms" were described occasionally in patients with arterial hypertension. ${ }^{12}$ The diameter of these "miliary" aneurysms usually ranged between 0.3 and $0.9 \mathrm{~mm}$. By definition, the perforating artery aneurysm described in our patient clearly differs from such "microaneurysms". Small aneurysms of perforating arteries might represent a congenital abnormality. Alternatively, autoimmune processes could be involved in their pathophysiology.

JOANNA D KOWNACKI

Department of Neurosurgery

LUCA REMONDA

Department of Neuroradiology

NELSON GODOY

JOACHIM K KRAUSS

Department of Neurosurgery, Inselspital,

University of Berne, 3010 Berne,
Switzerland

Correspondence to: Dr Joachim K Krauss, Department of Neurosurgery, Inselspital, University of Berne, 3010 Berne, Switzerland. Telephone 0041316323444 ; fax 0041313822414.

1 Russell RWR. Observations on intracerebral aneurysms. Brain 1963;86:425-42.

2 Leeds NE, Goldberg HI. Lenticulostriate artery abnormalities. Radiology 1970;97:377-83. 